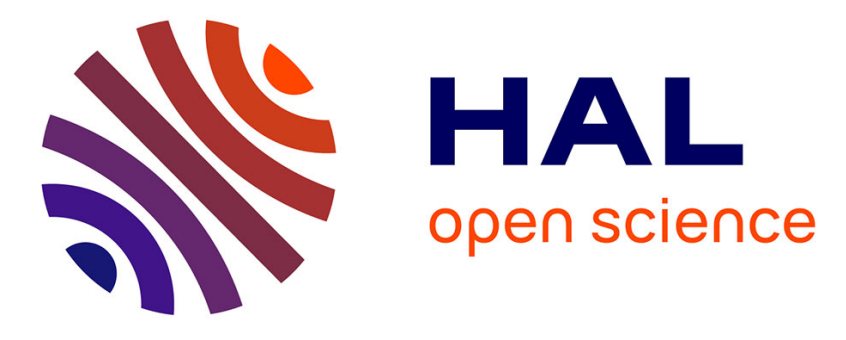

\title{
Quelle politique éducative pour la mise en place du e-learning dans l'enseignement supérieur : Le cas de la Chine et de la France
}

Micheline Marie-Sainte

\section{- To cite this version:}

Micheline Marie-Sainte. Quelle politique éducative pour la mise en place du e-learning dans l'enseignement supérieur : Le cas de la Chine et de la France. Terminal. Technologie de l'information, culture \& société, 2016, 118, 10.4000/terminal.1490 . hal-01653233

HAL Id: hal-01653233

https://hal-univ-tlse2.archives-ouvertes.fr/hal-01653233

Submitted on 4 Dec 2017

HAL is a multi-disciplinary open access archive for the deposit and dissemination of scientific research documents, whether they are published or not. The documents may come from teaching and research institutions in France or abroad, or from public or private research centers.
L'archive ouverte pluridisciplinaire HAL, est destinée au dépôt et à la diffusion de documents scientifiques de niveau recherche, publiés ou non, émanant des établissements d'enseignement et de recherche français ou étrangers, des laboratoires publics ou privés. 


\title{
QUELLE POLITIQUE EDUCATIVE POUR LA MISE EN PLACE DU E- LEARNING DANS L'ENSEIGNEMENT SUPÉRIEUR : Le cas de la Chine et de la France
}

\author{
Micheline Marie-Sainte \\ LISST
}

\section{Introduction}

L'introduction du numérique dans le système éducatif a bouleversé les pratiques et reconfiguré le marché de l'éducation et de la formation. L'arrivée des moocs et du buzz qu'elle génère en est une des dernières manifestations. Si l'introduction des technologies de l'information et de la communication dans l'éducation a d'abord visé l'amélioration pédagogique des enseignements, elle a été ensuite présentée comme un moyen de diminuer les dépenses liées à la formation (réduction des coûts, suppression de postes de fonctionnaires) pour les Etats et augmentation des recettes (ouverture à de nouveaux marchés).

Ainsi de nombreux établissements d'enseignement supérieur à l'échelon mondial qu'ils soient publics ou privés se sont emparés de ces nouvelles modalités de formation dans la perspective de toucher de nouveaux publics contraints pour la plupart (professionnels en exercice, étudiants distants ne pouvant avoir accès normalement à la formation en présentiel, étudiants étrangers).

Si les initiatives émanaient au départ principalement d'acteurs isolés, les Etats dans un contexte de globalisation (internet abolit les frontières), face à la concurrence internationale se sont mobilisés et ont été amenés à s'interroger sur le type de politique éducative à mettre œuvre pour étoffer leur offre de formation en e-learning. Les réponses n'ont pas été homogènes. Nous proposons dans cet article d'explorer les politiques mises en œuvre par deux Etats : la Chine et la France. Elles seront analysées au travers des processus de construction de leur offre de formation en matière de diplômes nationaux ainsi que des formations proposées.

Dans un premier temps nous exposerons les principes sur lesquels se fonde la politique éducative, puis nous exposerons la méthodologie mise en œuvre et les éléments recueillis en matière d'offre de formation. Pour aboutir à une présentation contrastée de deux types de politiques éducatives.

La mise en place d'une politique d'introduction et de mise en œuvre du e-learning peut s'appuyer sur : les trois grands principes qui fondent classiquement les politiques éducatives. Il s'agit de savoir quelles filières ouvrir, à quel niveau et quel en sera l'effectif. On distingue ainsi :

- le principe du besoin de main-d'œuvre

- le principe du rendement social

- le principe de la demande sociale

A ces trois principes de départ apparait un quatrième plus transversal : celui de l'équité.

Le principe du besoin de main consiste pour le système éducatif ou de formation à répondre au besoin de main-d'œuvre de l'économie. Ce principe s'appuie sur l'hypothèse d'une relation formation-emploi forte, de type mécaniste. Cette hypothèse a donné lieu à un certain nombre de modèles macroéconomiques de besoin de main d'œuvre utilisés dans le cadre de la planification indicative française pour les différents plans qui se sont succédés du IVème qui a débuté en 1962 au IXème de 1984 à 1988. Ces modèles reposent sur l'hypothèse de fonctions de production 
macro-économique à coefficients techniques fixes, à partir desquels sont déterminés les effectifs de chaque catégorie de main-d'œuvre nécessaire au fonctionnement de l'économie. Cependant, la plus grande critique faite à ce type de modèle repose sur une vision adéquationniste de la relation formation-emploi et on peut citer en cela l'ouvrage coordonné par Lucie Tanguy paru en 1986 « l'introuvable relation formation-emploi ».

Dans le cas du principe du rendement social l'éducation est considérée comme investissement collectif. Comme tout investissement, il génère des coûts et des bénéfices pour la collectivité. On associe un taux de rendement social qui guidera le choix de telle ou telle filière. On se place ici résolument dans la perspective du capital humain (Becker 1964, Mincer 1962). Il s'agira de prendre en compte dans sa totalité les coûts supportés par la collectivité ainsi que les bénéfices engendrés.

- Pour les coûts on rajoutera dans cette perspective ce qui n'avait pas été pris en compte dans les coûts individuels :

- les coûts en personnel éducatif et non éducatif

- les achats de livres et de matériel pédagogiques

- les dépenses courantes de chauffage, d'éclairage, d'entretien

- l'amortissement des bâtiments

- les transferts de l'Etat en termes de bourses allouées aux étudiants

- les déperditions et redoublement (comme indiqué par Psacharopoulous et Woodhall 1988)

- Pour les gains, en plus de la rémunération obtenue par les individus, il faudra ajouter :

- les recettes fiscales supplémentaires liées au revenu plus élevé (impôt sur le revenu) et aussi la TVA (plus le revenu est élevé, plus l'individu consomme, plus il y a production de TVA)

- plus généralement les externalités non monétaires mais là on se heurte à la difficulté de les évaluer en terme de monnaie.

On peut alors s'interroger sur la validité d'un tel indicateur compte-tenu du fait qu'il existe un temps plus ou moins long entre le moment où la formation est décidée et le moment où les individus arrivent effectivement sur le marché du travail - les choses peuvent changer. De plus, il n'est pas dit que favoriser qu'une catégorie d'emplois n'occasionnera pas des déséquilibres, avec par exemple, in fine des conséquences sur le niveau des salaires des sortants (forte concurrence). Et quel fonctionnement pour une société où il n'y aurait que des docteurs...

Dans le cadre du principe de la demande sociale, l'institution tente de répondre aux souhaits des apprenants ou des familles sans se soucier du devenir des apprenants sur le marché du travail. On est plus dans ce cas sur un positionnement «formation » comme service de consommation, c'est-à-dire, service auquel est affecté une utilité. Les familles vont chercher à maximiser leur utilité (satisfaction) sous une contrainte de budget et «d'aptitude » à suivre la formation. Cela nécessite du système de formation une réactivité certaine car les demandes exprimées des familles ne sont connues dans le meilleur des cas que 6 mois avant l'entrée en formation (procédure de préinscription post bac actuellement en France) voire la veille de la rentrée dans certaines filières.

Le principe basé sur l'équité peut s'envisager sous deux angles : un géographique (accès distant) et l'autre social, c'est-à- dire d'accès aux personnes « empêchées » (handicapés, en emploi).

L'équité en terme d'accès géographique renvoie à la couverture territoriale. Une fois les filières de formation déterminées, la politique éducative peut aussi se donner pour objectif de toucher un public particulier qui jusque-là n'était pas accessible.

Le plus grand challenge du e-learning est avant tout de pouvoir couvrir un territoire donné : l'accès à internet reste la première condition de sa mise en oeuvre. Cependant avec la 
généralisation de l'internet par le satellite cette difficulté s'estompe, en dépit des coûts qui sont significatifs. On estime en France, à environ 400 euros (prix 2013) l'investissement nécessaire comprenant l'acquisition du matériel (modem et parabole) auquel il faut ajouter le coût humain lié à l'installation (environ 150 euros). Il y faut ajouter le coût de l'abonnement qui lui reste relativement abordable (30 euros environ par mois) ${ }^{1}$. Cette technologie permet une connexion stable, et fiable sur l'ensemble d'un territoire. Les zones dites blanches ou grises comme c'est souvent le cas dans les zones rurales peuvent ainsi être desservies. Il apparaît intéressant de constater que les pays où le e-learning a connu un fort développement ont un point commun : ils sont constitués le plus souvent de vastes territoires : c'est le cas des Etats-Unis, de l'Australie, de la Chine, de l'Inde ou encore du Canada.

\section{L'accès aux personnes en emploi et impulsion du changement}

Outre l'accès à la formation à des personnes éloignées géographiquement, l'e-learning peut toucher aussi des publics en emploi. Cette mesure peut permettre au sens où l'entend Amartya Sen de contribuer à ce que ces personnes puissent mener la vie professionnelle de leur choix sans que cela représente un coût insurmontable pour elles dans le sens où elles n'auraient pas à choisir entre se former ou travailler.

En France, la problématique du choix entre formation et maintien de rémunération n'est pas insurmontable puisqu'un certain nombre de dispositions du droit social permet aux individus de suivre une formation sans perte de revenu. C'est le cas pour les temps de formations financés par les Fongecifs ou autres OPCA (organismes paritaires collecteurs agréés). Cependant, si ces dispositions existent, leurs mises en œuvre ne sont pas automatiques. Elles nécessitent l'accord des instances pour les unes et de l'employeur pour les autres. L'individu n'est pas l'unique décisionnaire. Le choix du e-learning apparaît comme un véritable espace de liberté : l'individu peut seul décider de se former sans attendre d'autorisation autre.

De plus, ce qui pourrait apparaître comme une stricte mesure d'équité, peut être un élément central dans la mise en œuvre rapide et ciblée d'une politique de formation en vue de l'acquisition de nouvelles compétences ou la mise en œuvre de nouvelles pratiques (exemple de l'agro-écologie dans l'enseignement agricole français). Dans un contexte général d'accélération des processus, d'obsolescence des savoirs due notamment à l'usage des TIC à tous les niveaux de la société, il apparaît vital, voir essentiel de se former rapidement. Le e-learning permet de répondre à la problématique de la formation rapide en poste. Les personnes en emplois sont déjà insérées, il n'y a pas de déperdition de «capacités » due à un mauvais appariement entre formation et emploi (la relation formation/emploi n'est pas automatique, il peut y avoir des décalages en terme de niveau, de spécialité, de territoire - Tangy L. 1986, Vernière M. 1997), ni de temps de latence dû au processus d'insertion. L'impact de la formation de ces personnes sera d'autant plus fort qu'elles occupent une place clé dans la société permettant l'amorce d'un changement au niveau d'une institution, ou d'un pays.

\footnotetext{
${ }^{1}$ pour aller plus loin, voir le comparatif de prix : http://www.internetparsatellite.net/usr/www internetparsatellite_net/documents/comparatif-par-prix.html - site consulté en novembre 2013
} 


\section{Méthodologie}

Pour identifiée les politiques éducatives mises en place en matière de e-learning nous avons effectué un recensement exhaustif de l'offre de formation en France et en Chine.

\section{L'offre de formation chinoise}

Durant l'été 2011, nous nous sommes adressés à une étudiante chinoise Jun WU du master 2 «Intelligence économique » de l'Université Toulouse 1, pour réaliser un recueil d'informations sur le e-learning en Chine.

Ce travail a donné lieu à la constitution d'une grande base de données faisant suite à une veille sur internet sur les formations à distance diplômantes (promotion d'automne 2011). Les caractéristiques suivantes ont été retenues :

- l'institution

- la région

- le domaine de formation

- le volume horaire

- le type de public

- les points d'enseignement

- le tutorat

- le niveau

- le type de diplôme

Toutes les informations à l'exception du prix ont été trouvées sur le site officiel des universités, dans son règlement ou ses modalités d'inscription.

Cette grande base de données provient de l'agrégation de trois sous-ensembles qui portent respectivement sur (le système éducatif chinois, de l'enseignement supérieur fait la différence en terme de niveau entre les diplômes «bac+3 licence» considérés comme professionnels et « licence ou master » qui sont eux considérés comme des diplômes « pleinement universitaires»):

- les FOAD visant les étudiants inscrits dans le deuxième cycle chinois (diplôme délivré en «Bac+3 ou Licence ») ;

- les FOAD visant les étudiants dans au moins un niveau de troisième cycle chinois, c'est-àdire correspondant au diplôme Licence ou Master

- Les prix proposés par les points d'étude présents dans les différentes régions. La plupart des prix des formations ont été obtenus par téléphone et messagerie électronique avec un responsable des agences locales. Les autres ont été trouvés dans des organisations reconnues dans le domaine de la formation à distance.

Nous avons effectué une vielle sur le développement de la formation à distance assortie d'une recherche d'informations contextuelles sur le niveau actuel du marché éducatif global et le niveau de vie en Chine. Ces informations ont été recueillies selon plusieurs voies : 
- les informations en chinois par les moteurs de recherche comme Google, Baidu², les informations en Français ou en Anglais, par les moteurs de recherche comme Netvibes ${ }^{3}$ avec comme mot clé « e-Learning en Chine».

- l'abonnement à 9 flux RSS ${ }^{4}$ provenant des revues ou journaux internationaux sur l'e-Learning en Digimind Evolution", filtrés automatiquement avec des requêtes comme "China » ou «Chine ».

\section{L'offre de formation française}

Le travail lié au recensement de l'offre de formation française a été réalisé par un groupe de quatre étudiants du master 2 intelligence économique de l'Université Toulouse 1: Benjamin Dambrine, Thi Phan Hang N'guyen, Thui Van Vun, Jun Wu.

Il est possible que certaines formations n'apparaissent pas et ce pour différentes raisons : la date de création (ou de début de communication) de la formation est postérieure à la date de la recherche (octobre 2010 à mars 2011), la formation a été exclue du champ de la recherche car celle-ci n'utilisait pas ou trop peu les TIC pour pouvoir être entièrement à distance. Lors de la recherche, cette formation devait ne pas être visible sur internet (ni dans les annuaires, ni sur les sites spécialisés ou universitaires).

Ce travail de recensement a été réalisé à partir des informations présentes sur le net. Les champs suivants ont été renseignés :

- référencé par l'AUF ou non

- niveau de la formation

- recherche ou professionnel

- domaine

- intitulé de la formation

- université/Ecole (nom de l'établissement d'origine)

- prix de la formation

- type de public (quand c'est spécifié : formation continue, formation initiale, formation prise en charge par une entreprise)

- tutorat

- nom de la plate-forme numérique

- synchrone/asynchrone

- volume horaire

- langue d'enseignement

- lien source

Une base comprenant 128 diplômes a pu être constituée.

Nous proposons une comparaison France Chine pour l'année 2011. La Chine apparaît avec un mode opératoire de type «top-down» agissant en double détente: d'une part par une

\footnotetext{
2 Baidu est le plus grand moteur de recherche en Chine après Google, il est sorti sur le marché chinois en 2010.

${ }^{3}$ Netvibes est un portail Web français personnalisable, représentatif de ce qu'on appelle le Web 2.0. Il a été lancé le 15 septembre 2005 et est gratuit www.Netvibes.com

${ }^{4}$ RSS (Really Simple Syndication) est un format de description de données qui facilite la diffusion de fils d'informations, de façon automatisée, à l'intention des internautes, et qui favorise la syndication de contenus en permettant à d'autres sites de republier facilement tout ou partie de ces données. (source : http://www.01net.com)

${ }^{5}$ L'outil de veille stratégique reconnu en France. La version Digimind Evolution est gratuite. Elle s'utiliser avec un compte provisoire pour les étudiants.
} 
différentiation du prix des formations en fonction des territoires de destination, d'autre part par un choix de contenu de formation qui procède d'un choix délibéré de type de capacités à développer sur le territoire.

En France la politique de la mise en œuvre du e-learning apparait plus souple avec une utilisation de «l'appel à projet financé par le ministère de l'enseignement supérieur » et où le choix des formations en e-learning relève de la seule volonté des établissements qui jouissent d'une certaine autonomie.

\section{Résultats}

\section{La Chine}

La Chine, à bien des égards nous a paru exemplaire dans sa politique de mise en œuvre du elearning. Nous l'avions repéré lors d'un premier survey effectué par les étudiants du master Intelligence économique de Toulouse 1. C'est pourquoi, nous avons demandé à une des étudiantes, Wu Jun, d'effectuer un travail d'approfondissement sur la Chine.

La Chine s'est lancé il y a maintenant, plus d'une dizaine d'années dans une politique volontariste de mise en œuvre d'une offre de formation universitaire en e-learning. Ce sont les principales caractéristiques de cette offre que nous présentons à la suite.

A la fin des années 1990, le gouvernement chinois décide, à travers le projet $211^{6}$ d'initier une politique de formation visant à privilégier le développement socio-économique s'appuyant sur des universités bénéficiant des standards internationaux en matière de recherche. Au départ prévu pour une centaine d'universités, ce sont 69 d'entre-elles, qui seront positionnées avec une offre de formation représentant des disciplines « clés », dites " spécialités extraordinaires ».

Mais avant d'en venir au système de formation proprement dit, il nous a semblé important de rappeler quelques chiffres qui caractérisent la Chine.

Tableau 1 : La Chine en chiffre (source Etat du monde)

\begin{tabular}{|l|c|c|c|c|c|c|}
\hline \multicolumn{1}{|c|}{ CHINE } & & $\mathbf{1 9 8 7}$ & $\mathbf{2 0 0 0}$ & $\mathbf{2 0 0 9}$ & $\mathbf{2 0 1 0}$ & $\mathbf{2 0 1 1}$ \\
\hline \multicolumn{1}{|c|}{ DÉMOGRAPHIE } & & & & & & \\
\hline Population & (million) & 1092 & 1269 & 1335 & 1341 &.. \\
\hline Densité & $\left.(\text { hab./km })^{2}\right)$ & 114 & 132,2 & 139,1 & 139,8 &.. \\
\hline Indice de fécondité (ISF) & $($ enf./femme) & 3 & 1,75 & 1,62 & 1,6 &.. \\
\hline Mortalité infantile & $(\%$ ) & 34 & 26 & 21,3 & 20,8 &.. \\
\hline Espérance de vie & $($ année) & 69 & 71,2 & 73 & 73,2 &.. \\
\hline INDICATEURS SOCIOCULTURELS & & & & & & \\
\hline Nombre de médecins & $(\%$ hab.) & 1 & 1,64 & 1,42 &.. &.. \\
\hline Analphabétisme (hommes) & $(\%)$ &.. & 4,9 &.. & 2,9 &.. \\
\hline Analphabétisme (femmes) & $(\%)$ &.. & 13,5 &.. & 8,7 &.. \\
\hline Scolarisation 2e degré & $(\%)$ & 35 & 62,1 & 80,1 & 81,2 &.. \\
\hline Scolarisation 3e degré & $(\%)$ & 3 & 8 & 24,3 & 25,9 &.. \\
\hline
\end{tabular}

\footnotetext{
${ }^{6}$ Lancé durant la période de 1996-2000, pour développer 100 universités ou spécialités extraordinaires en Chine. La définition en Anglais http://en.wikipedia.org/wiki/Project_211
} 


\begin{tabular}{|l|c|c|c|c|c|c|}
\hline Téléviseurs & (\%ohab.) & 87 & 304 &.. &.. &.. \\
\hline Accès à Internet & (\%ohab.) &.. & 17,9 & 289,8 & 343,8 &.. \\
\hline \multicolumn{1}{|c|}{ ECONOMIE } & & & & & & \\
\hline PIB total (PPA) & $($ milliard\$) & 679 & 3015 & 9066 & 10128 & 11300 \\
\hline Croissance annuelle PIB & $(\%)$ & 12 & 8,4 & 9,2 & 10,4 & 9,2 \\
\hline PIB par habitant (PPA) & $(\$)$ & 621 & 2379 & 6792 & 7550 & 8382 \\
\hline Investissement (FBCF) & $(\%$ PIB) & 32 & 34,1 & 46 & 45,4 &.. \\
\hline Taux d'inflation & $(\%)$ & 7 &.. &.. & 3,3 & 5,4 \\
\hline Population active & $($ million) &.. & 724 & 794 & 800 &.. \\
\hline Taux de chômage & $(\%)$ & 2 & 3,1 & 4,3 &.. &.. \\
\hline Énergie (taux de couverture) & $(\%)$ & 104 & 97,2 & 92,4 &.. &.. \\
\hline Dette extérieure totale & $($ milliard\$) & 35 & 145,3 & 432,2 & 548,6 &.. \\
\hline Service de la dette/Export & $(\%)$ & 10 & 9,1 & 2,8 & 3,3 &.. \\
\hline
\end{tabular}

On notera l'augmentation importante de part de la population scolarisée en troisième degré (enseignement supérieur) qui passe de $8 \%$ en 2000 à près de $26 \%$ en 2010, soit une augmentation de plus du triple en seulement dix ans.

\subsection{Le système éducatif chinois}

\section{Généralités}

La scolarité obligatoire est gratuite et dure 9 ans ( 6 ans en primaire et 3 ans au collège). Après, elle devient payante.

Le système d'enseignement secondaire chinois est un peu complexe. Les écoles sont partagées en deux catégories : les écoles professionnelles et les lycées généraux. Les écoles professionnelles ont pour but de donner aux apprenants des compétences professionnelles. Elles offrent des cours dans les domaines tels que l'industrie, les services, les affaires et l'agriculture. Le lycée général, quant à lui se consacre à l'objectif de préparer les étudiants à passer favorablement le GAOKAO général $^{7}$ (équivalent du baccalauréat français) et à entrer dans une université chinoise pour obtenir un diplôme de Licence Bac+4 (suivre une formation présentielle durant 4 ans). Le GAOKAO est un concours incontournable pour suivre directement la formation de Licence.

Par ailleurs, il existe également un GAOKAO professionnel $^{8}$ qui correspond à un concours d'entrée à un institut d'enseignement supérieur spécialisé. Il s'adresse aux étudiants de l'enseignement professionnel secondaire. Dès qu'ils l'ont réussi, ils peuvent suivre une formation présentielle en 3 ans et obtenir un diplôme de niveau Bac $+3^{9}$ (Dazhuan) dans une université ou un institut éducatif professionnel du supérieur. Ce diplôme plutôt pratique est considéré d'un niveau inférieur à celui de la licence $\mathrm{Bac}+4$ et a donc un seuil d'entrée inférieur (il est moins sélectif).

Les étudiants issus du lycée général peuvent suivre ce type de formation si leur résultat au GAOKAO général ne leur a pas permis d'être admis par les instituts d'enseignement supérieur général. Dans ce système éducatif, un titulaire du diplôme de niveau Bac+3 peut continuer d'étudier dans une formation de niveau Licence (études en 2 ans supplémentaires) ou Master (études en 5 ans supplémentaire).

\footnotetext{
${ }^{7}$ Le concours national chinois organisé par le M.O.E du 6-8 juin de chaque an

${ }^{8}$ Le concours spécialisé pour l'école professionnelle passé en Avril de chaque an.

${ }^{9}$ Ce diplôme professionnel se prépare en 3 ans mais d'un niveau inférieur à la Licence Bac+4
} 


\section{Figure 1 : Positionnement des bases de données dans le système éducatif chinois}

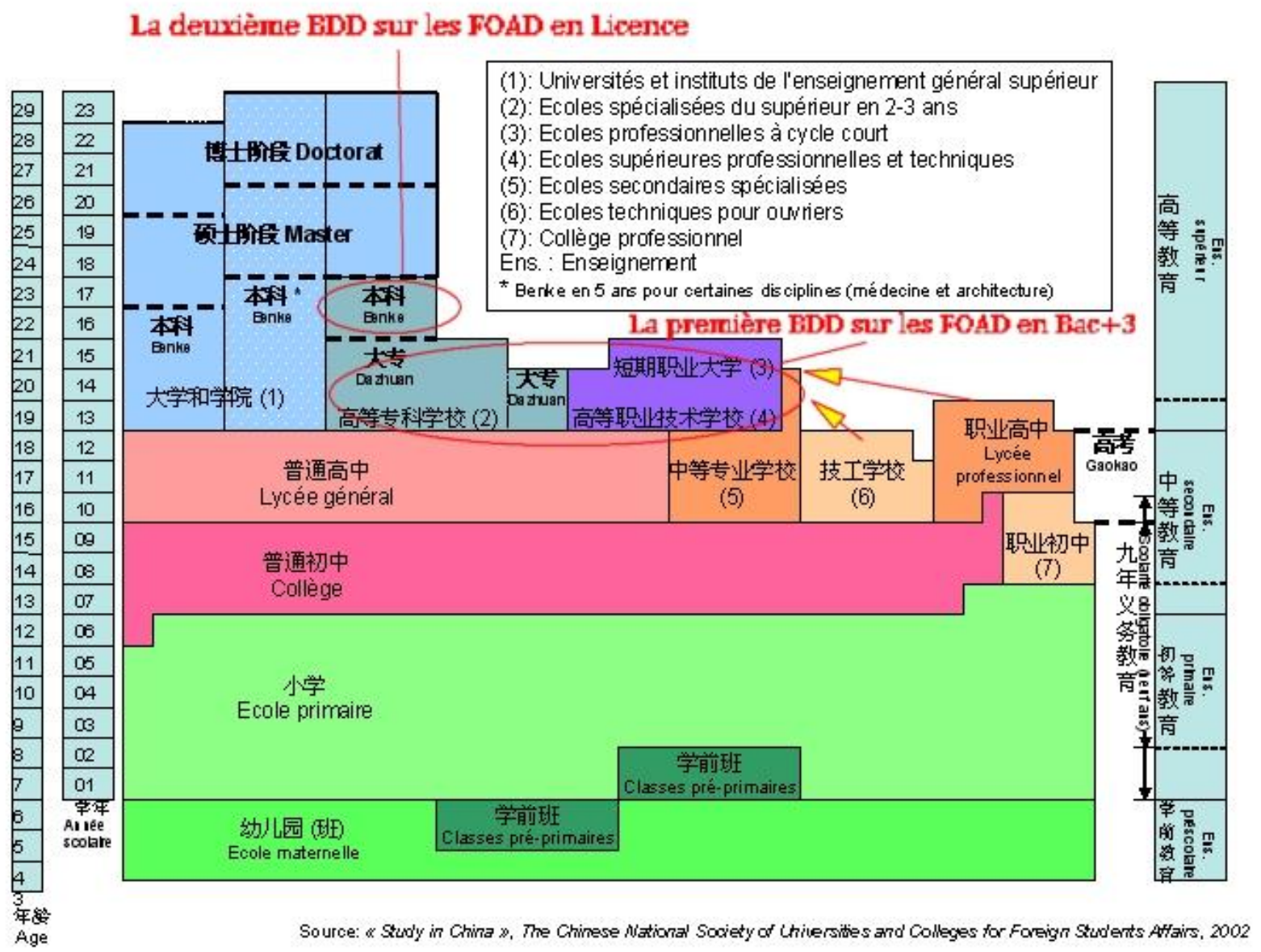

Presque toutes les universités chinoises contraignent les étudiants à vivre sur le campus. Durant leurs années d'enseignement supérieur, les élèves contraints de payer non seulement les frais d'inscription et les frais de scolarité correspondant à la formation qu'ils ont choisi, mais aussi l'hébergement (généralement de 3200 à 6000 Yuan chacune des 4 années scolaires en Licence).

L'augmentation progressive des effectifs d'étudiants a fait ressortir certaines limites telles que l'insuffisance des infrastructures et la distribution inégale des ressources éducatives. En outre, avec une augmentation du coût de la vie en milieu urbain, les frais de scolarité et les frais d'hébergement se sont accrus d'année en année. Simultanément, l'écart de prix entre les différentes villes s'est creusé. Certains élèves dans les zones reculées ont vu également croître leur coût d'étude dans une autre ville.

L'enseignement à distance pouvait jouer un rôle " gagnant-gagnant » bénéfique à l'université et aux étudiants. Il assurait les ressources pédagogiques, permettait de soulager la pression démographique à laquelle était soumises les institutions, permettait de résoudre les problèmes liés à l'espace et à la distance pour les étudiants lointains ou les travailleurs.

\section{Principaux acteurs du e-learning en Chine :}

Le projet " OpenEducation » a été lancé officiellement par China Central Radio and TV University en décembre 2002, avec l'autorisation du M.O.E10 et la collaboration du groupe OPENEDU. L'OpenEducation est également un organisme qui participe à la construction et au 
développement du e-Learning moderne en Chine. Cet organisme offre un ensemble de services aux institutions éducatives, comme la gestion des inscriptions, la gestion de la scolarité, le suivi des étudiants et enseignants, l'organisation des épreuves, jusqu' à la construction d'une plateforme et de la transmission de ressource. Actuellement, L'OpenEducation travaille pour 40 universités nationales et plus de 300 institutions de formation connues. Il a établi plus de 1400 points d'études et environ 400 centres de formation. Ce système offre des formations à distance et diplômantes de niveaux différents, ainsi que des formations professionnelles pour plus de 5 000000 d'étudiants. 11

Xuexigang est un système professionnel de service en éducation à distance, c'est un site influent créé par le magazine « Distance Education in China » pour toute la société et plus de 15000000 apprenants en ligne. Il offre des informations et formations sur l'éducation à distance, en plus d'une plateforme pour communiquer le fruit de la recherche. En outre, il permet aux institutions éducatives d'augmenter leur part de marché par la voie d'une procédure de "préinscription ». Il est considéré comme un des médias en ligne les plus influents dans l'industrie éducative.

Les universités offreuses de formation en e-learning sont au nombre de 69 (y compris une Open University) qui représentent les institutions supérieures les plus importantes. Elles sont reconnues comme «université pilote d'enseignement à distance » par le Ministère de l'éducation de la République populaire de Chine (M.O.E) et se situent dans 15 provinces et 4 municipalités (Beijing, Shanghai, Chongqing, et Tianjin).

\section{Tableau 2 : Répartition des 69 universités pilotes dans les provinces (base de données des FOAD chinoise juin 2011)}

\begin{tabular}{|l|c|}
\hline Province & Nombre d'universités \\
\hline Anhui & 1 \\
\hline Chongqing & 2 \\
\hline Fujian & 2 \\
\hline Gansu & 1 \\
\hline Guangdong & 3 \\
\hline Heilongjiang & 2 \\
\hline Henan & 1 \\
\hline Hubei & 5 \\
\hline Hunan & 2 \\
\hline Jiangsu & 3 \\
\hline Jilin & 2 \\
\hline Liaoning & 4 \\
\hline Pékin & 19 \\
\hline Shaanxi & 3 \\
\hline Shandong & 2 \\
\hline Shanghai & 7 \\
\hline Sichuan & 7 \\
\hline Tianjin & 2 \\
\hline Zhejiang & 1 \\
\hline TOTAL & 69 \\
\hline
\end{tabular}

On notera que 19 de ces universités sont localisées à Beijing qui constitue le centre économique et politique du pays. Grâce à cet avantage, ces universités ont fait fonction de "défricheurs »

\footnotetext{
11 http://www.open.com.cn/guanyu/index.htm (en Chinois)
} 
dans le développement des FOAD en Chine. En seconde position arrivent, Shanghai et Sichuan qui apparaissent comme deux régions remarquables avec 7 universités présentes. Shanghai, ville internationale a bénéficié d'un développement économique rapide du fait de son positionnement de ville commerciale avec l'étranger, et Sichuan possède un grand nombre d'universités en raison du déplacement des ressources éducatives vers l'intérieur des terres avant la révolution. C'est un centre majeur de l'enseignement supérieur chinois.

Les universités en Chine sont classées par niveau. Ce classement dépend de plusieurs éléments : la réputation, la qualité de la formation, la compétence des enseignants, le nombre de demandeurs et le niveau des infrastructures etc.

Nous avons dressé un tableau récapitulant le niveau des 69 universités pilotes dans l'enseignement en ligne. Plus le nombre d'étoiles est important, plus l'université possède de notoriété.

Dans le tableau ci-après, figure les noms des universités en abrégé, les dénominations complètes et la liste des universités sont mises en annexe.

Tableau 3 : Notoriété des universités (source : base de données des FOAD chinoises juin 2011)

\begin{tabular}{|l|l|}
\hline Notoriété de l'université & Nom de l'université \\
\hline$\star \star \star \star \star \star$ & Tsinghua University, Peking University \\
\hline$\star \star \star \star \star$ & BNU, BUAA, Fudan, Tongji, SYSU, HIT, SCU, XJTU, etc. \\
\hline$\star \star \star \star$ & BUPT, BIT, BJU, NEU, UESTC, USTC, SWJTU, ECUST, etc. \\
\hline$\star \star \star$ & BFSU, BUCM, CPU, SWNU, SYTU, CMU, ZZU etc. \\
\hline$<\star \star \star$ & BLCU, NEAU, DUFE, FJTU, SICAU \\
\hline
\end{tabular}

\subsection{L'offre de formation en e-learning}

La répartition du niveau des FOAD est très hétérogène. En mai 2011, il y avait 2135 formations à distance diplômantes proposées par les universités chinoises.

Sur le tableau qui suit, on observe que $43.42 \%$ des formations sont du niveau Bac +3 , et $56.44 \%$ sont de niveau licence (pour mémoire le niveau licence est équivalent à Bac+4). Il y a 1205 formations au niveau Licence, parce que la demande au niveau de ce diplôme est importante. En effet, les demandeurs se partagent en deux groupes : les jeunes qui ont réussi au GAOKAO national et les titulaires d'un diplôme Bac+3.

Tableau 4 : Offre de formation par niveau de formations (source : base de données des FOAD chinoises juin 2011)

\begin{tabular}{|l|l|l|}
\hline Niveau & Nombre & $\mathbf{\%}$ \\
\hline Bac+3 & 927 & $43,42 \%$ \\
\hline Licence & 1205 & $56,44 \%$ \\
\hline Master & 3 & $0,14 \%$ \\
\hline TOTAL & 2135 & $100 \%$ \\
\hline
\end{tabular}

En outre, il y a peu de formations au niveau Master par rapport au système d'enseignement à distance en France : seules trois formations de ce niveau étaient offertes en 2011 par l'Université 
de Tsinghua. Ce sont les Master 2 Technologie de l'information, Master 2 Management des entreprises et Master 2 Code civil et droit des affaires.

\section{Carte 1 : Répartition des FOAD en Chine par région (Licence) (base des données des FOAD chinoises juin 2011)}

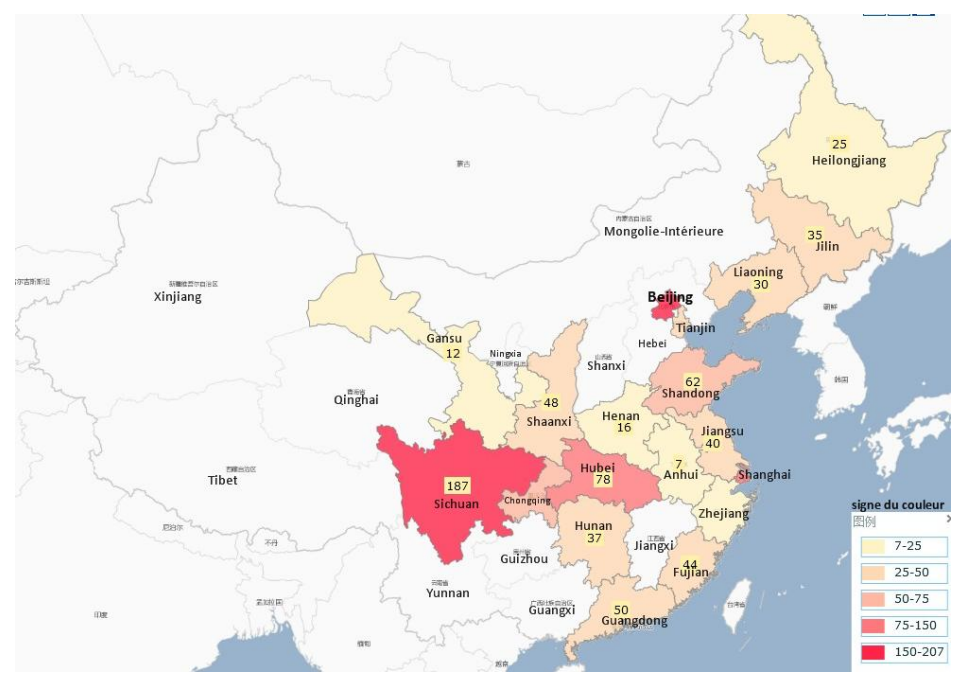

Cette carte a été réalisée par le site «Shujuditu».http://www.shujuditu.com/

L'origine de l'offre de formation à distance est concentrée dans l'Est, le Centre, le Sud-ouest et le Sud-est de la Chine. Par contre, il existe très peu de FOAD dans les régions de l'Ouest et du Nord. Deux éléments semblent expliquer la répartition de l'offre de formation en e-learning en Chine : le facteur démographique et le développement économique.

En ce qui concerne le facteur démographique, la carte de la répartition de la population en Chine $^{12}$, qui suit, montre la densité de la population qui est symbolisée par l'intensité de la couleur. On peut retrouver facilement les régions de l'Est et du Sud-ouest (la province du Sichuan en particulier) qui sont les régions les plus peuplées et qui disposent d'un grand nombre de FOAD. Par contre, la région de l'Ouest et la région du Nord, sont vastes et peu peuplées, les infrastructures éducatives y sont difficiles à implanter à cause des contraintes liées au transport, aux conditions géographiques et aux facteurs politiques (en particulier pour la région tibétaine et la région autonome de Xinjiang).

\section{Carte 2 : Répartition de la population en Chine (récupérée juin 2011)}

\footnotetext{
${ }^{12}$ La source de la carte : http://bbs.godeyes.cn/
} 


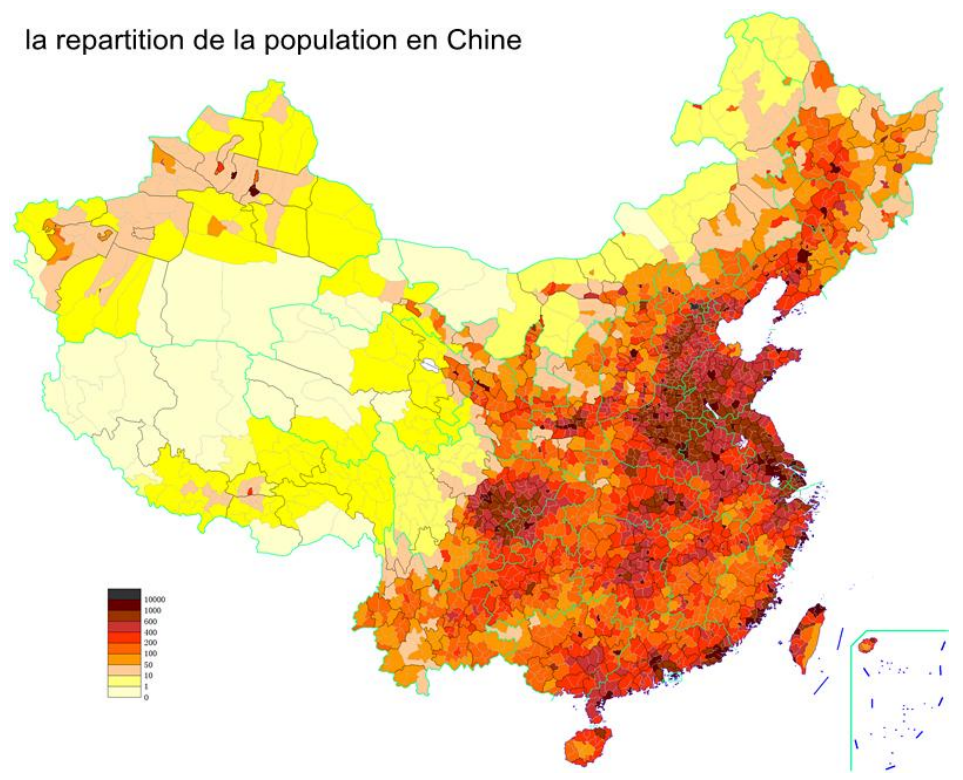

Le développement économique apparaît comme une autre condition importante permettant le déploiement de l'offre de formation en e-learning. En effet, il assure l'infrastructure technologique (électricité, réseau) pour la mise en œuvre de l'enseignement à distance ainsi que la ressource humaine formée nécessaire. La région du Sud-est et la région de l'Est ont une position importante dans le développement économique en Chine. Avec la politique de l'«Economie ouverte sur les régions côtières », les régions comme Guangdong, Fujian, Shanghai, Zhejiang et Tianjin, sont des pionniers dans le développement de l'enseignement à distance en Chine.

Depuis 1999, le projet « démarrage expérimental de l'enseignement à distance » a été initié avec la ratification du M.O.E chinois. C'est le départ du e-learning développé dans les instituts d'enseignement supérieur. Pendant 12 ans, le nombre total des participants de l'enseignement moderne en ligne a atteint environ 10 millions. ${ }^{13}$. En outre, des dizaines de milliers de points d'enseignement ont été établis dans les villes et les villages afin de participer au transfert des ressources éducatives vers les zones rurales et vers l'ouest de la Chine.

\section{Domaine}

Dans l'offre de formation en e-learning, ce sont les domaines «économie-gestion-commerce » et «science technologie et ingénierie » qui ressortent principalement. Plus de $2 / 5$ des formations concernent une activité économique et commerciale, et environ $1 / 3$ des formations des domaines scientifiques et technologiques.

\section{Graphique 1 : Répartition des formations par domaine (base de données des FOAD chinoises juin 2011}

13 La nouvelle a été exposée dans la conférence de presse par le M.O.E le 15 avril 2011. 

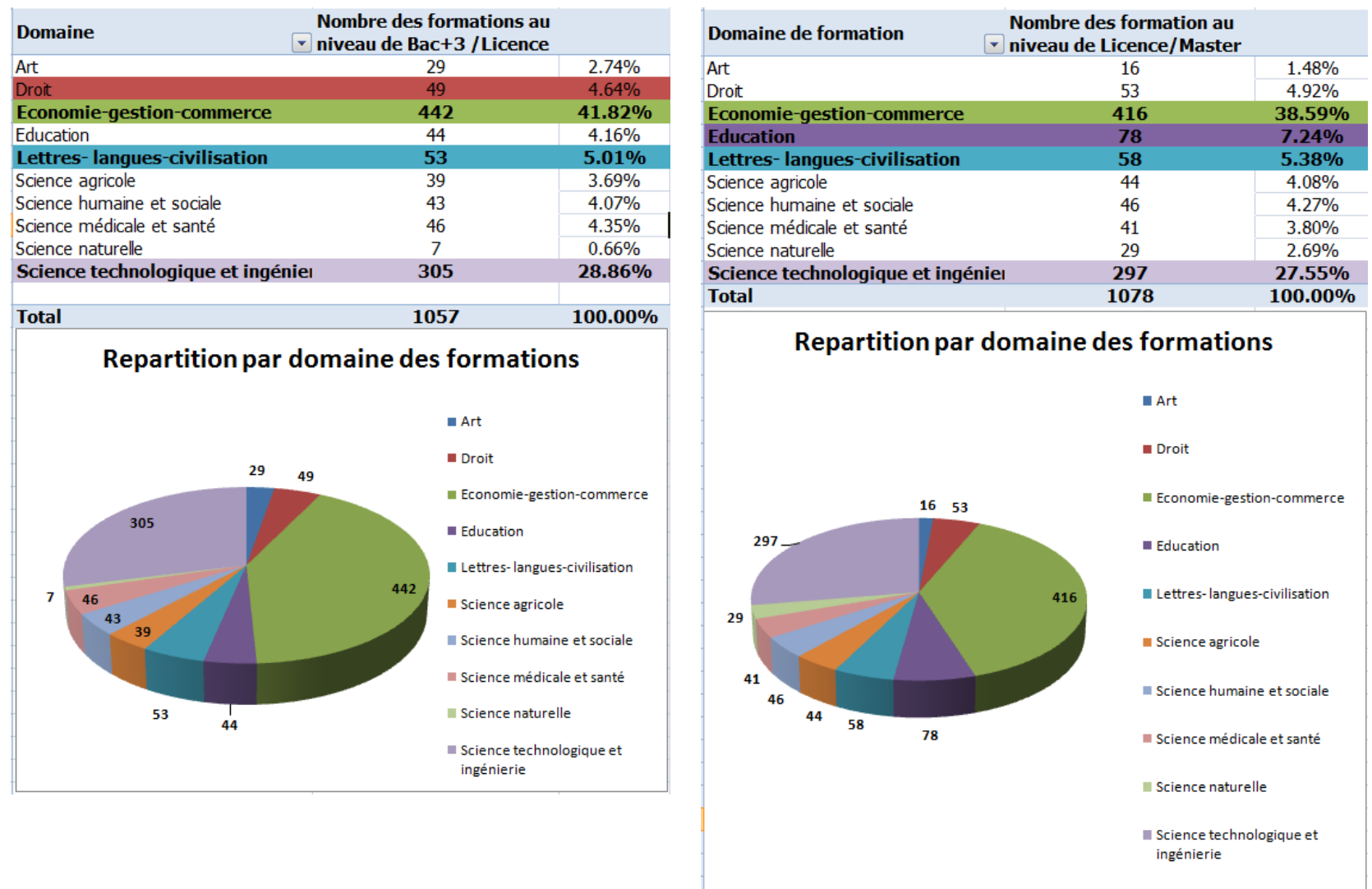

La répartition des diplômes par domaines dans les deux bases de données reste sensiblement la même à part celui relatif à l'éducation où l'offre apparaît plus importante au niveau licence/master.

\section{Type de public}

Le public ciblé est très large, il peut s'agir d'étudiants, de personnes en reprise d'études ou de travailleurs.

Tableau 5 : Type de public pour les niveaux bac+3/licence (base de données des FOAD chinoises juin 2011)

\begin{tabular}{|l|r|r|}
\hline Type de public & Nombre & $\mathbf{\%}$ \\
\hline FC & 354 & $33,49 \%$ \\
\hline FC (titulaire du certificat médical professionnel) & 37 & $3,50 \%$ \\
\hline FC/FI & 656 & $62,06 \%$ \\
\hline FC/FI (avec une base de musique) & 3 & $0,28 \%$ \\
\hline FC/FI (titulaire du certificat médical professionnel) & 7 & $0,66 \%$ \\
\hline TOTAL & 1057 & $100 \%$ \\
\hline
\end{tabular}

Tableau 6 : Type de public pour le niveau licence/master (base de données des FOAD chinoises juin 2011)

\begin{tabular}{|l|r|r|}
\hline Type de public & Nombre & $\mathbf{\%}$ \\
\hline FC & 307 & $28,48 \%$ \\
\hline FC (avec de l'expérience professionnelle) & 123 & $11,41 \%$ \\
\hline $\begin{array}{l}\text { FC (titulaire du certificat médical professionnel avec de l'expérience } \\
\text { professionnelle) }\end{array}$ & 31 & $2,88 \%$ \\
\hline
\end{tabular}




\begin{tabular}{|l|r|r|}
\hline FC (titulaire du certificat professionnel) & 8 & $0,74 \%$ \\
\hline $\begin{array}{l}\text { FC (titulaire du diplôme de licence avec 3 ans d'expérience } \\
\text { professionnelle) }\end{array}$ & 3 & $0,28 \%$ \\
\hline FC/FI & 592 & $54,92 \%$ \\
\hline FC/FI (avec une base de musique) & 3 & $0,28 \%$ \\
\hline FC/FI (titulaire du certificat médical professionnel) & 9 & $0,83 \%$ \\
\hline FC /FI (titulaire du certificat professionnel) & 2 & $0,19 \%$ \\
\hline TOTAL & 1078 & $100 \%$ \\
\hline
\end{tabular}

Au niveau bac+3, environ $62 \%$ des formations sont ouvertes à tous les public (FC/FI). Certaines le sont uniquement pour les travailleurs (environ 33.5\%). En outre, il existe des conditions particulières qui en limitent l'accès pour certains domaines spécialisés comme la médecine et la musique: des qualifications et expériences professionnelles sont nécessaires pour suivre ces formations.

Pour le niveau licence, tous les public (FC/FI) ont également un large choix aux formations (environ 55\% des formations), contre environ $40 \%$ des formations qui sont ouvertes uniquement aux travailleurs.

\section{Les points d'enseignement}

Comme pour le modèle de l'AUF, les universités établissent de nombreux points d'enseignement (équivalent aux campus numériques) dans les provinces et villes chinoises grâce à des partenariats avec des institutions éducatives locales. Certaines collaborent avec des organisations spécialisées dans l'e-learning comme l'OpenEducation et le XUEXIGUANG, qui disposent de leurs propres agences dans les régions.

Les points d'enseignements offrent un certain nombre de service, allant de l'administration pédagogique comme la sélection des étudiants, le tutorat, l'organisation des examens, à la mise en place des infrastructures telle que la disposition des ordinateurs et la préparation des salles.

La mise en place d'une institution permettant la mutualisation des formations en e-learning rend le système de gestion plus aisé. A titre d'exemple en 2011, l'Open Education avait reçu mandat de 40 universités nationales et plus de 300 institutions de formation connues. Il avait ainsi pu établir plus de 1400 points d'enseignement et environ 400 centres de formation qui offraient des FOAD diplômantes de niveaux différents, ainsi que des formations professionnelles à plus 5000 000 apprenants $^{14}$.

\section{Prix des formations}

Collecter tous les prix pratiqués par les 69 universités était difficile. Afin de réduire les erreurs nous avons travaillé à partir d'un échantillon : parmi les 69 universités, nous avons retenus les universités où un "prix/crédit» de la formation avait été trouvé. Cela concerne 45 universités sur 69. Puis, dans chacune de ces universités, nous n'avons retenu qu'une seule formation pour chaque domaine (car le prix par crédit dans un même domaine de formation est le même).

Le travail suivant a ensuite été d'effectuer un découpage des points d'études en cinq zones :

- Beijing,

\footnotetext{
${ }^{14} \mathrm{http}: / /$ www.open.com.cn/guanyu/index.htm (en Chinois)
} 
- Shanghai,

- la zone côtière,

- la zone du développement central

- la zone de l'Ouest

Pour chaque région, un prix moyen par crédit a été calculé en différenciant les niveaux des universités. Les prix des formations ont été calculés en euros car cela permet la comparaison entre tarifs chinois et tarifs européens $(1$ euro $=10$ Yuan $){ }^{15}$

\section{Tableau 7 : Prix des formations en fonction des universités d'origine et du positionnement des points d'enseignement (base de données des FOAD chinoises juin 2011)}

\begin{tabular}{c|c|c|c|c|c|}
\hline $\begin{array}{c}\text { Classe de des universités } \\
\text { chinoises (en étoile) }\end{array}$ & Pékin & Shanghai & $\begin{array}{c}\text { Régions } \\
\text { côtières }\end{array}$ & $\begin{array}{c}\text { Régions de dé } \\
\text { veloppement } \\
\text { Centrale }\end{array}$ & $\begin{array}{c}\text { Régions de } \\
\text { I'Ouest }\end{array}$ \\
\hline$<\star \star \star$ & 9.375 & 10.909 & 9.563 & 7.031 & 6.344 \\
\hline$\star \star \star$ & 10.045 & 10.923 & 9.234 & 7.845 & 6.730 \\
\hline$\star \star \star \star$ & 10.364 & 11.625 & 9.844 & 7.761 & 7.027 \\
\hline$\star \star \star \star \star$ & 11.723 & 12.804 & 10.277 & 7.958 & 7.315 \\
\hline$\star \star \star \star \star$ & 20.625 & 23.125 & 21.250 & 20.000 & 16.500 \\
\hline
\end{tabular}

Les prix des FOAD sont proposés par crédit. Le prix du crédit varie en fonction du niveau des régions où les points d'enseignement se situent, en fonction la notoriété de l'université où les formations sont proposées, en fonction du domaine de formation.

Le prix par crédit d'une FOAD est décidé en fonction du niveau de prix de la région. En Chine, les centres économiques comme Beijing, Shanghai et la région côtière sont plus coûteux que les autres régions. De plus, pour la région de l'Ouest comme Qinghai, Shaanxi, Gansu, le gouvernement chinois a mis en œuvre une stratégie "de développement de l'Ouest » 16 pour permettre d'équilibrer le développement dans les différentes régions. Dans le tableau, on peut voir qu'entre deux prix, correspondant à Shanghai et la région de l'Ouest, l'écart de prix par crédit atteint environ 5 euros.

Le prix par crédit est fonction du niveau général de l'université, d'après le classement des universités chinoises, plus le nombre d'étoiles de l'université est important, plus le prix par crédit d'une formation proposée est élevé. Ainsi, un prix moyen par crédit des formations proposées par les universités en six étoiles, peut être approximativement deux fois plus élevé que celui d'une université moins étoilée. Dans les régions du développement central et la région de l'Ouest, cet écart est à peu près de trois fois. (16.5 pour une université à six étoiles contre 6.3 pour une à moins de trois étoiles)

Le prix par crédit est différent par domaine. En général, une formation en économie-gestioncommerce est relativement moins coûteuse qu'une en science technologique et ingénierie. Les formations en Art et en Science médicale et santé sont plus chères, car elles sont rares et spécialisées. Pour certaines universités spécialisées dans le domaine agricole, les formations sont moins coûteuses parce qu'elles ciblent souvent des étudiants dans les régions rurales qui n’ont pas les moyens de suivre une formation onéreuse.

\footnotetext{
15 Une échange approximatif entre le monnaie euro et RMB

16http://www.observateurocde.org/news/fullstory.php/aid/419/D_E9veloppement_r_E9gional_en_Chine_html
} 
En Chine, la sélection d'entrée dans une université renommée est sévère. Les lycéens doivent passer le GAOKAO et obtenir la note correspondante aux attentes des différentes universités. Bien que le nombre d'inscriptions prévues ait augmenté d'année en année, les universités reconnues sont encore difficiles à atteindre pour les jeunes. Par exemple en 2009, le taux d'entrée à une université connue (université du projet 211) à Pékin était seulement de $24 \%$, même si le taux de recrutement dans l'enseignement supérieur atteignait 72.3\%.17

A l'inverse, le seuil d'entrée dans le système d'enseignement à distance chinois est plus accessible, la sélection y est moins sévère. Cela permet ainsi, à de nombreux étudiants de poursuivre dans une université où ils n'avaient pas pu entrer après le GAOKAO.

De plus, il apparaît que le prix des formations en e-learning est similaire à celle des formations en présence. Certaines formations à distance proposent même un prix inférieur à celles en présentiel, notamment dans les régions de l'Ouest et les régions centrales de la Chine. L'enseignement à distance tel qu'il est pratiqué permet ainsi de résoudre le problème du paiement des formations pour les étudiants ayant peu de ressource.

La mise en place du e-learning en Chine a permis de résoudre un double défi : celui de l'augmentation du niveau de qualification de la main d'œuvre avec son corollaire, la massification de l'entrée dans l'enseignement supérieur et la nécessité d'étendre cette montée en qualification à l'ensemble de son territoire. Le fait que les formations à distance aient un prix différent en fonction de la région de l'apprenant en témoigne. Une remarque importante doit aussi être mentionnée: cette volonté d'extension de l'accès aux diplômes s'adresse à tous les types de publics, formation initiale ou formation continue avec toutefois un accès plus étendu pour ce dernier public.

\section{La France}

\subsection{Politique publique et le e-learning, rappel}

Le développement des formations en e-learning a souvent coïncidé avec les campagnes impulsées par la puissance publique.

Rappelons qu'en France, pour impulser l'usage des TIC dans l'éducation, le ministère de l'enseignement supérieur, dès la fin des années 90 met en place des contrats avec les universités pour l'implantation des TIC, c'est le projet (PAGSI : plan d'action gouvernementale pour la société de l'information) en 1997.

En 2000, les campus numériques : sur trois ans l'Etat, par le biais du ministère de l'enseignement supérieur et de la Datar financent des appels à projet pour un montant de quelques 21,34 millions d'Euros. L'objectif est de constituer une offre «marchande » de formation structurée, de qualité et compétitive, pouvant faire concurrence à l’hégémonie anglo-saxonne.

A partir de 2007, concernant les Universités Numériques Thématiques (UNT), toujours sur le même mode opératoire d'appel à projet, le ministère sélectionne et finance les projets retenus.

\footnotetext{
17 taux d'entrée à une université connue a Pékin en 2009 http://www.ce.cn/xwzx/kj/201105/03/t20110503_22395949.shtml (en Chinois)
} 
En termes d'efficacité on peut être circonspect compte-tenu du peu d'usage que ces ressources ont au sein de la communauté des étudiants (voir plus haut).

En 2013 le projet France Université Numérique a à sa disposition 12 millions d'euros disponibles pour les projets de MOOC ayant répondu aux appels à projet.

\subsection{Etat des lieux des FOAD françaises}

La France propose des formations entières constituées de cours. Ainsi, 128 formations ont été répertoriées dont 25 font parties de l'AUF en mars 2011. Ces formations sont donc uniquement basées sur le territoire français. Nous déterminerons, un profil type de ces FOAD.

Tableau 8 : Offre de formation en e-learning par région administrative (base de données des FOAD françaises mars 2011)

\begin{tabular}{|l|c|}
\hline \multicolumn{1}{|c|}{ région } & nombre de FOAD \\
\hline Alsace & 8 \\
\hline Aquitaine & 5 \\
\hline Auvergne & 6 \\
\hline Basse Normandie & 1 \\
\hline Bourgogne & 1 \\
\hline Bretagne & 2 \\
\hline Champagne Ardennes & 7 \\
\hline Franche-Comté & 12 \\
\hline Haute Normandie & 12 \\
\hline Ile-de-France & 2 \\
\hline Languedoc Roussillon & 1 \\
\hline Limousin & 13 \\
\hline Lorraine & 3 \\
\hline Midi-Pyrénées & 13 \\
\hline Nord-Pas-de-Calais & \\
\hline Pays de la Loire & \\
\hline Picardie & \\
\hline Poitou-Charentes & \\
\hline Provence-Alpes-Côte d'Azur & \\
\hline
\end{tabular}




\begin{tabular}{|l|c|}
\hline Rhône-Alpes & 13 \\
\hline Total & 128 \\
\hline
\end{tabular}

Quand on place l'offre de formation en e-learning sur la carte de la France en fonction de son université d'origine, elle apparaît pour le moins inégalement répartie.

\section{Carte 3 : Offre de formation en e-learning par région (base des données des FOAD françaises mars 2011)}

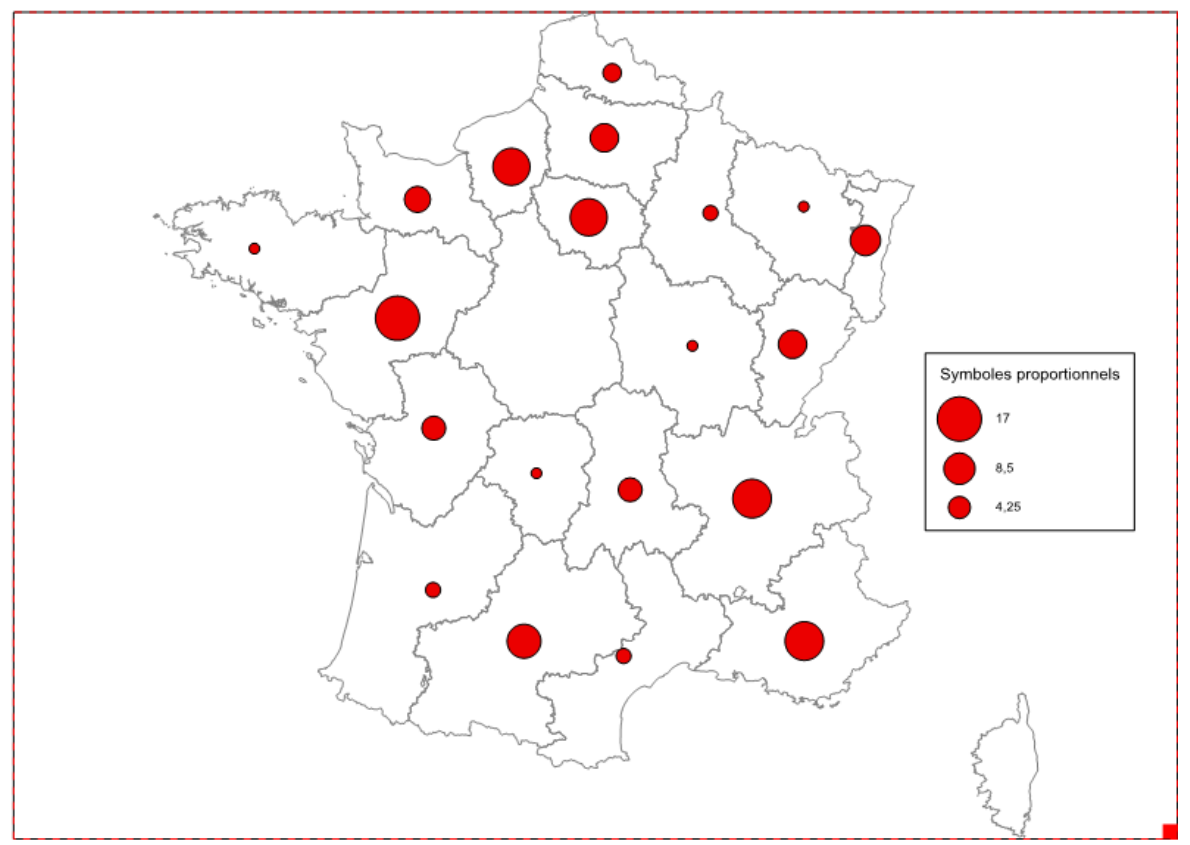

La dimension du disque est proportionnelle au nombre de FOAD dans chacune des régions.

Une remarque : la diagonale (du nord-est au sud-ouest) semble correspondre à la France du « vide ». En effet, l'offre de formation y est anecdotique. De même, le nombre de FOAD proposées n'est pas proportionnelle au nombre d'étudiants : la région parisienne, premier site universitaire national et Toulouse, deuxième site universitaire ne sont pas classés en tête.

Trois régions se distinguent : Les pays de la Loire, la région Rhône Alpes et la région ProvenceAlpes-Côte d'Azur.

Enfin, il est intéressant de souligner que les DROM-COM ${ }^{18}$ sont totalement absents de la formation à distance.

Cette carte à une portée toute relative et n'est en aucun cas faite pour montrer le marché de la FOAD. En effet, le principe même du e-learning est de casser les distances et de permettre de toucher un public de l'autre côté de la planète sans autres frontières que celle de la langue. Elle est simplement utile pour déterminer si certaines régions sont plus impliquées que d'autres dans la mise en œuvre d'une politique de l'université en matière de formations à distance. Elle est d'ailleurs à mettre en regard avec celle représentant la répartition des étudiants sur le territoire :

\footnotetext{
18 Départements et Régions d'Outre-Mer \& Collectivités d'Outre-Mer
} 


\section{Carte 4 : Etudiants inscrits dans l'enseignement supérieur en 2010-2011}

Source :

http://cache.media.enseignementsuprecherche.gouv.fr/file/Etat_du_Sup_Rech/18/3/EESR_2011_209183.pdf

19

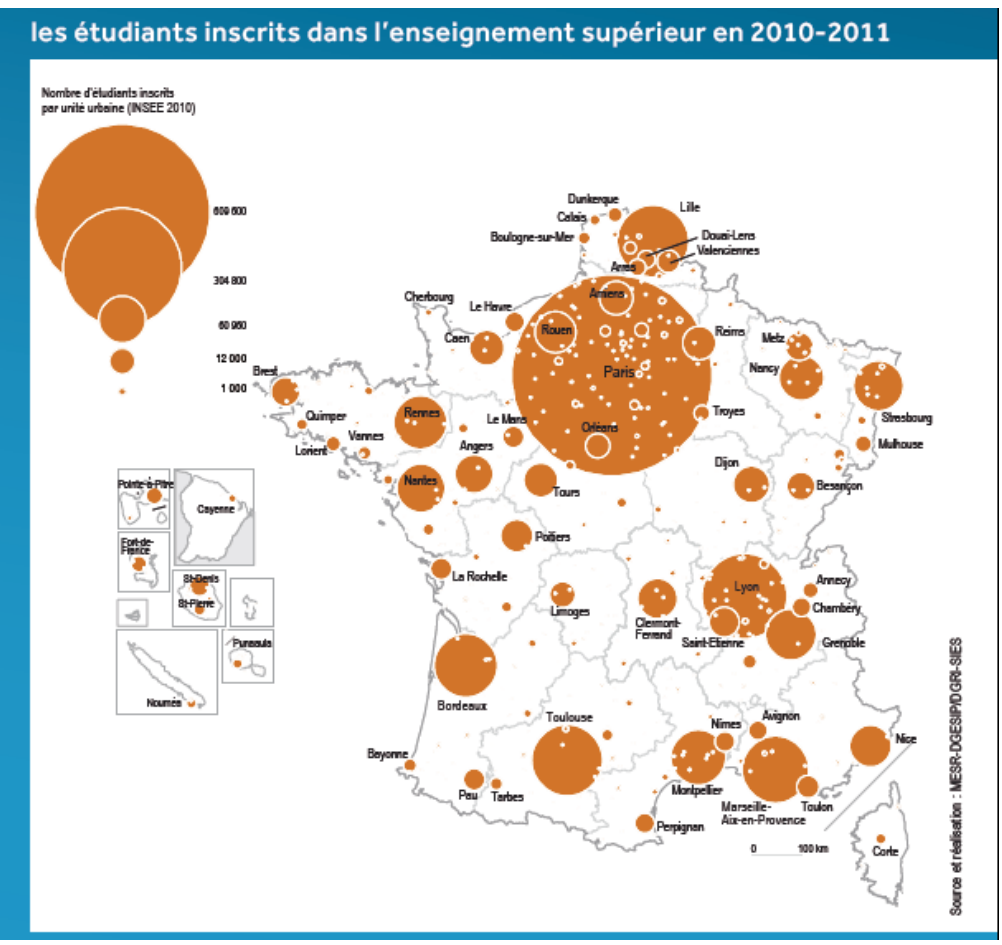

On a sensiblement la même distribution des principaux pôles, même si pour le cas de l'île de France l'offre en e-learning est loin d'être proportionnelle au nombre d'étudiants. C'est certainement là un effet «capitale » qui fait d'une part qu'avec ses treize universités, l'île de France apparaît comme un territoire bien couvert et d'autre part, que sa position de « métropole » a un effet d'attraction pour bon nombre d'étudiants qui peuvent ainsi bénéficier de tous les avantages liés au statut d'étudiants dans une grand ville.

Nous avons voulu entrer plus dans le détail et voir si il pouvait y avoir une corrélation entre le nombre d'étudiants d'une université et la présence ou non d'e-learning dans l'offre de formation de celle-ci et plus généralement quels pouvaient être les facteurs d'ouverture de FOAD.

Tableau 9 : Effectif d'étudiants en 2009-2010 par université et offre de formation en elearning en mars 2011 (base de données des FOAD françaises)

\begin{tabular}{|l|r|r|}
\hline & $\begin{array}{l}\text { Nombre de } \\
\text { FOAD en } \\
\text { UNIVERSITES }\end{array}$ & $\begin{array}{l}\text { Effectifs } \\
\text { d'étudiants } \\
\text { en 2009-201020 }\end{array}$ \\
\hline Université d'Avignon et des pays de Vaucluse & 2 & 6457 \\
\hline Université du Maine: Le Mans & 13 & 10111 \\
\hline Université Blaise pascale Clermont Ferrand 2 & 5 & 13880 \\
\hline Université de Limoges & 1 & 14191 \\
\hline $\begin{array}{l}\text { Université de Versailles Saint-Quentin-en- } \\
\text { Yvelines }\end{array}$ & 3 & 14623 \\
\hline Université Paul Valery Montpellier 3 & 2 & 15768 \\
\hline Université Paris 3 & 1 & 16973 \\
\hline
\end{tabular}

19 consulté en juin 2013 


\begin{tabular}{|l|r|r|}
\hline Université Haute-Bretagne, Rennes 2 & 1 & 17004 \\
\hline Université de Cergy-Pontoise & 1 & 17338 \\
\hline Université Pierre Mendés-France Grenoble 2 & 11 & 17826 \\
\hline Université Victor Segalen Bordeaux 2 & 2 & 17895 \\
\hline Université Toulouse 1 Capitole & 7 & 18267 \\
\hline Université Charles de Gaulle - Lille 3 & 1 & 18357 \\
\hline Université de Lille 1 & 2 & 18439 \\
\hline Université de Franche-Comté Besançon & 7 & 19884 \\
\hline Université de Provence Aix Marseille 2 & 2 & 20489 \\
\hline Université de Reims Champagne Ardennes & 2 & 21873 \\
\hline Université Paris 8 & 5 & 21972 \\
\hline Université Aix-Marseille 1 & 7 & 22294 \\
\hline Université Jules Verne - Picardie & 7 & 22448 \\
\hline Université Toulouse 2 le Mirail & 3 & 23001 \\
\hline Université de Poitiers (IAE) & 5 & 23919 \\
\hline Université de Rouen & 12 & 24201 \\
\hline Université de Caen Basse-Normandie & 6 & 24253 \\
\hline Université de Nice Sophia-Antipolis & 2 & 26484 \\
\hline Université de Bourgogne - Dijon & 1 & 26897 \\
\hline Université Lumière Lyon 2 & 1 & 27405 \\
\hline Université Paris X Nanterre & 1 & 29459 \\
\hline Université Pierre et Marie Curie Sorbonne & 1 & 29560 \\
\hline Université de Nantes & 4 & 33017 \\
\hline Université Claude Bernard Lyon 1 & 1 & 34364 \\
\hline Université de Strasbourg & 8 & 42243 \\
\hline Université de Nancy & 1 & 48549 \\
\hline Ua majorité des universités ayant un & 2 & 040258 \\
\hline
\end{tabular}

La majorité des universités ayant une offre en e-learning s'approche ou dépasse les 15000 étudiants à part deux exemples flagrants : le Mans et Avignon. En effet, on peut penser qu'il faille un minimum de moyens financiers et humains pour mettre en place du e-learning. Et les universités ont des moyens financiers attribués par l'Etat en fonction du nombre d'étudiants (repéré à n-1). Ceci explique donc en partie à l'inverse, le nombre important de petites universités qui n'ont pas d'offre de formation en e-learning. En voici quelques unes :

Tableau 10 : Quelques universités peu importantes en effectif d'étudiants (chiffres DEPP 2009-2010)

\begin{tabular}{|l|l|}
\hline Universités et académies & Effectif d'étudiants \\
\hline Bordeaux 1 & 9005 \\
\hline Corse & 3714 \\
\hline Grenoble 3 & 5555 \\
\hline Nîmes & 3276 \\
\hline La Rochelle & 7162 \\
\hline Bretagne Sud & 8203 \\
\hline Le Havre & 6957 \\
\hline
\end{tabular}

Cependant l'effectif n'est pas la seule explication, loin s'en faut. Pour mémoire, nous avions mené une analyse, selon la méthodologie proposée par Michel Godet (2007) permettant de déterminer quelles étaient les variables motrices du développement des dispositifs de formation en elearning dans le système universitaire français. Nous vous proposons ici, quelques éléments clés de cette étude qui apporte un début de réponse. 
Un dispositif de formation ouverte et à distance est un système complexe (ensemble d'éléments en interaction), il s'est agit d'analyser la structure du système afin de comprendre son fonctionnement.

Les variables : "la formation et la qualification de la ressource humaine ", "la coordination externe» et "la volonté stratégique» apparaissent comme les variables endogènes clés du développement des dispositifs de formation en FOAD. C'est certainement de ce côté que se trouve l'explication de la présence de petites universités dans la liste de celles proposant de la FOAD, et inversement, l'absence de grosses. Il apparaît en effet que sans un minimum de qualification de la ressource humaine et sans une forte volonté du sommet stratégique (Président d'université, Directeur d'école, conseil d'administration), toute politique de développement à l'égard du elearning ne peut prendre corps.

\section{Niveau de formation}

L'offre de française, se situe le plus souvent au niveau au master 2 (M2) (environ 68\%), 32\% représentant l'offre au niveau licence. Les formations au niveau doctorat n'ont pas été repérées.

\section{Graphique 2 : Offre en terme de niveau de diplôme (base de données des FOAD françaises} mars 2011)

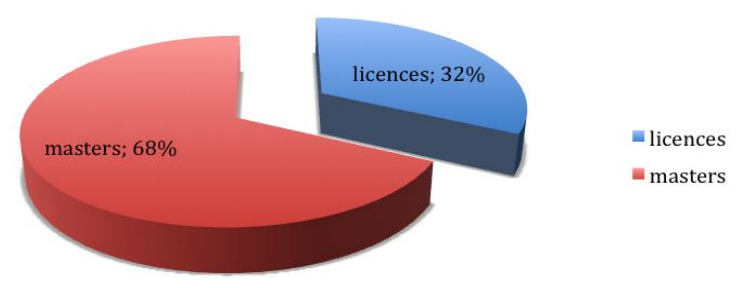

Brève note sur l'offre de formation au niveau maitrise (M1)

Après un aperçu de l'offre de FOAD de niveau M1 effectué grâce à divers annuaires de formation (Telesup, site de l'AUF...), le résultat donne un total d'environ 70 formations, 11 diplômantes (contre environ 86 en M2). A noter que ces formations sont bien souvent reliées à un master 2 particulier de manière directe (même intitulé ou descriptif de la formation).

Le fait d'avoir moins de M1 proposés que de M2 peut s'expliquer par le fait que la formation à distance est essentiellement axée vers un public en reprise d'étude ou professionnel qui ne peuvent donc assurer un acte continu de formation trop long. Il peut donc être compliqué pour ces derniers de s'impliquer durant au moins deux années entières dans une formation.

\section{Diplôme général ou professionnel}

Les formations dites professionnelles représentent $60 \%$ de l'effectif total des formations contre $20 \%$ pour celles axées sur la recherche et $20 \%$ pour celles identifiées comme généralistes.

\section{Graphique 3 : répartition entre diplômes professionnel/général ou recherche (base de données des FOAD françaises mars 2011)}




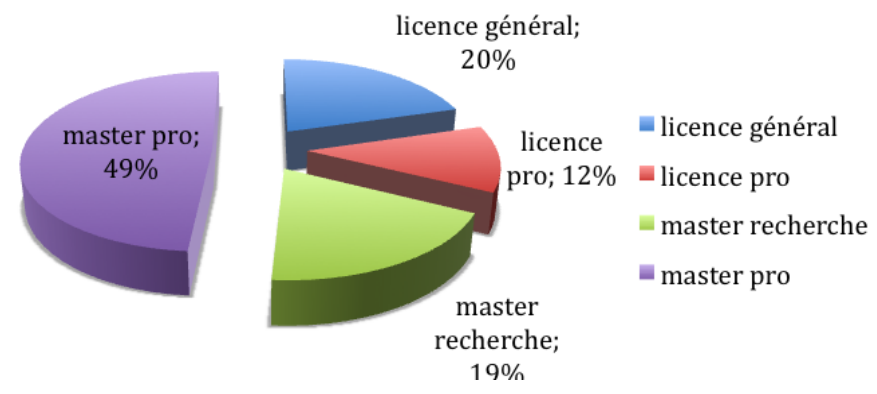

On voit bien sur ce graphique que les master pro correspondent à eux seuls à pratiquement la moitié des formations référencées.

\section{Champ disciplinaire}

Tableau 11 : Nombre de diplômes par domaine (base de données des FOAD françaises mars 2011)

\begin{tabular}{|l|r|}
\hline Domaine & Effectif \\
\hline Droit & 10 \\
\hline Eco gestion & 24 \\
\hline Education & 12 \\
\hline Informatique & 13 \\
\hline Lettre langue & 24 \\
\hline Science agricole & 2 \\
\hline Science dure & 11 \\
\hline Science humaine & 17 \\
\hline Science médicale & 10 \\
\hline Science technologie et ingénierie & 5 \\
\hline TOTAL & 128 \\
\hline
\end{tabular}

\section{Graphique 4 : Offre de diplômes par domaine disciplinaire (source base des données des FOAD françaises mars 2011)}

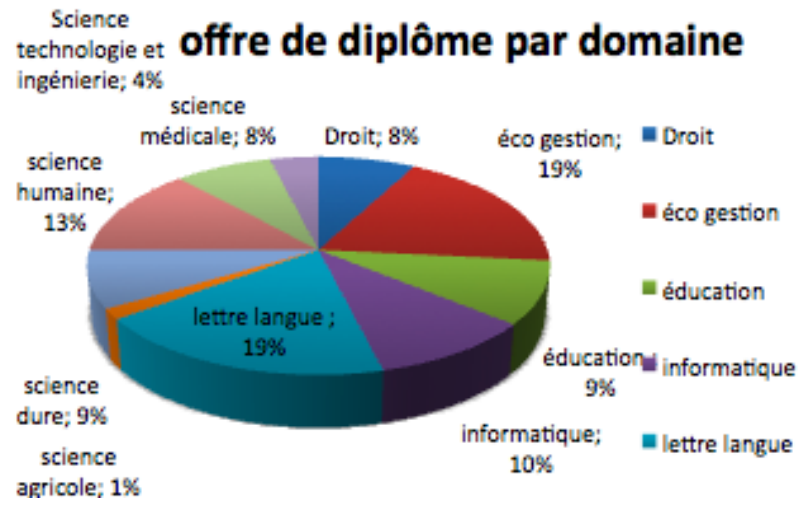

Dans les formules proposées en France, deux domaines d'activités ressortent principalement du panel tandis que les autres correspondent à des parts relativement homogènes. Ces deux domaines sont lettre-langue et économie-commerce-gestion. Ils représentent chacune 19\% du total des diplômes. Pour le premier domaine, celui des lettre-langue, cela peut s'expliquer en partie par le fait que ce type de formation n'est pas coûteux à mettre en place, le matériel pédagogique n'a pas besoin d'être lourd, et par le fait que cette forme de pédagogie convient bien à la demande. 
Pour l'autre domaine, celui de l'économie-gestion. On notera que 4 de ces formations proviennent de l'IAE de Poitiers et 6 de l'université Pierre Mendes France de Grenoble. Deux universités portent donc à elles seules plus de la moitié de l'offre dans ce secteur. Cela conforte l'idée qu'il semble qu'il se soit développé des expertises particulières au sein des universités, sachant que l'offre de formation "économie-gestion » est pratiquement proposée en présentiel par tous les sites universitaires.

On peut noter la grande différence de ces deux domaines : le premier semble difficile à appliquer au milieu professionnel tandis que le second est totalement intégré au monde de l'entreprise.

En affinant au niveau master on obtient la répartition qui suit :

\section{Graphique 5 : Répartition des masters pro par grand domaine (source base de données des FOAD françaises mars 2011)}

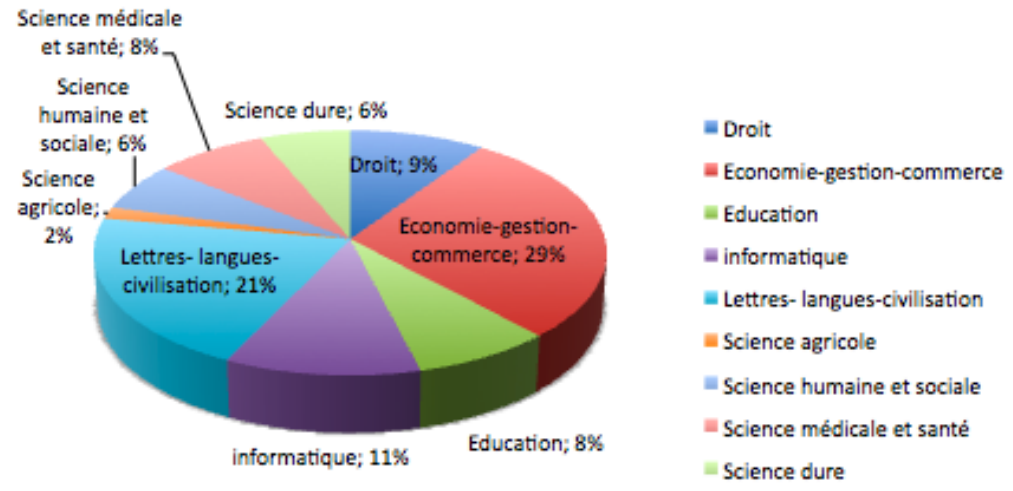

Le domaine économie-gestion-commerce creuse son écart avec $29 \%$ de l'effectif des masters pro par rapport au domaine lettre-langue-civilisation qui arrivait en première position à égalité avec lui.

Il semblerait que ce soit avant tout l'offre professionnelle, à destination des entreprises qui est motrice des formations en FOAD en France.

\section{Graphique 6 : répartition des masters recherche par domaine (source : base de données des FOAD françaises mars 2011)}

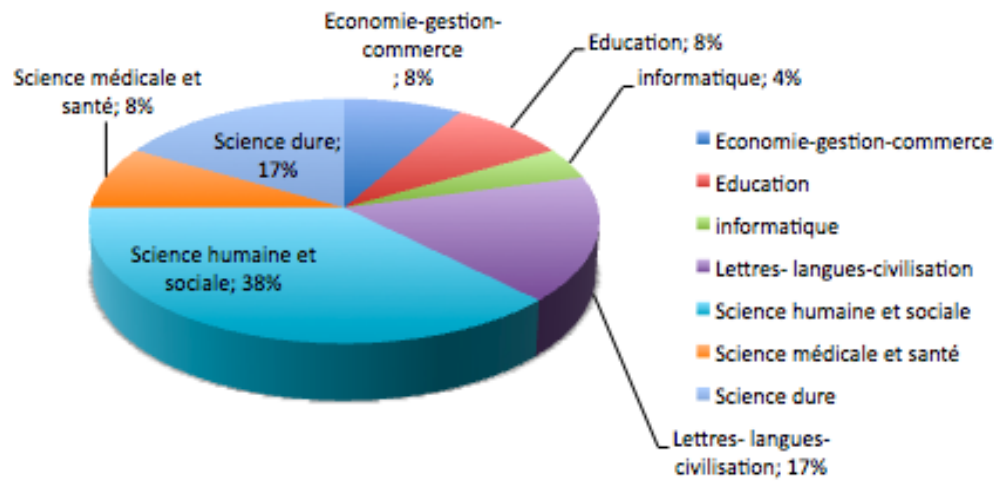

C'est le domaine science humaine et sociale qui arrive en tête de l'offre de formation en master recherche avec 9 diplômes sur 24, suivi par le domaine lettres-langues-civilisation. 


\section{Plateforme}

Les universités françaises communiquent très peu sur la plateforme utilisée : plus de $40 \%$ des formations ne communiquent pas. En ce qui concerne les informations disponibles, la plateforme Moodle se trouve en tête avec un peu moins du tiers des formations (38).

D'autres plateformes apparaissent comme Dokéos ou Acolad (mise au point par l'université de Strasbourg) mais cela ne concerne que quelques cas.

\section{Tutorat}

On peut constater que la quasi-totalité des formations propose un tutorat. Seules trois formations ne semblent pas en proposer. Cependant, ces résultats sont à manier avec précaution. En effet, ces trois formations ne fournissent aucune information permettant de déterminer si elles proposent un tutorat ou non. A défaut, nous avons donc décidé de les considérer comme non tutorées.

\section{Public}

Le constat montre encore une fois que les formations proposées sont ouvertes au plus large public possible. En effet, $94 \%$ de celles-ci sont ouvertes aux publics en formation initiale et $78 \%$ à ceux en formation continue.

Seules 8 formations sont ouvertes exclusivement à un profil de professionnel en formation continue tandis que pour la formation initiale, ce chiffre est plus que doublé. A savoir que $71 \%$ des formations sont ouvertes au moins à ces deux types de public.

\section{Synchrone ou asynchrone}

La technologie asynchrone semble être une nouvelle fois privilégiée pour profiter au maximum des avantages de l'enseignement à distance. Ainsi, toutes les formations utilisent au moins partiellement la technologie asynchrone tandis que 2/3 l'utilise exclusivement. Le reste met en place des formations utilisant les deux types de technologies : synchrone et asynchrone.

\section{Profil type}

L'offre de formation française en matière de FOAD ressemble à celle que l'on a pu identifier au niveau international: la communication se fait essentiellement au niveau des formations diplômantes et non de cours indépendants (même si certaines universités peuvent en proposer de façon plus spécifique).

La FOAD française est plutôt de niveau master et orientée professionnellement. Le domaine de formation peut être difficilement applicable au monde de l'entreprise comme lettre, langues et civilisation (19\%) ou au contraire totalement intégré comme Economie-Gestion-Management (19\%). Cette formation propose un fort tutorat dans le cadre d'une plateforme utilisant majoritairement une technologie asynchrone avec parfois des séances de regroupements virtuels. Cette dernière est mise en œuvre grâce à la plate-forme MOODLE malgré un manque de communication sur le LMS utilisé. La formation est ouverte à un maximum de public aussi bien en formation initiale qu'en formation continue. Enfin la langue de formation utilisée est en intégralité le français. 


\section{Les prix}

A noter que ces comparaisons ne sont valables que sur une période définie, c'est-à-dire celle du moment de l'enquête (année universitaire 2010-2011) car le monde du e-learning est en constante évolution.

Tableau 12 : Distribution des prix des formations en Euros (source base de données des FOAD françaises mars 2011)

\begin{tabular}{|c|c|c|c|c|c|}
\hline \multicolumn{6}{|c|}{ Moyennes } \\
\hline \multicolumn{3}{|c|}{ licence } & \multicolumn{3}{|c|}{ master } \\
\hline & $\begin{array}{l}\begin{array}{l}\text { Formation } \\
\text { complète }\end{array} \\
\end{array}$ & crédit & & $\begin{array}{l}\text { Formation } \\
\text { complète }\end{array}$ & crédit \\
\hline professionnel & 2419,21 & 40,32 & professionnel & 3013,23 & 50,22 \\
\hline Général & 1125,38 & 18,76 & Recherche & 2225,71 & 37,10 \\
\hline $\begin{array}{l}\text { Différence } \\
\text { pro/gen }\end{array}$ & $53 \%$ & & $\begin{array}{l}\text { Différence } \\
\text { pro/recher }\end{array}$ & $26 \%$ & \\
\hline total & 1689,89 & 28,16 & total & 2800,13 & 46,67 \\
\hline \multicolumn{6}{|c|}{ Ecarts de prix } \\
\hline \multicolumn{3}{|c|}{ Licence } & \multicolumn{3}{|c|}{ master } \\
\hline Prix haut & 7000 & 116,67 & Prix haut & 11000 & 183,33 \\
\hline Prix bas & 156 & 2,60 & Prix bas & 156 & 2,60 \\
\hline $\begin{array}{l}\text { Différence } \\
\text { Haut/bas }\end{array}$ & $X 44,87$ & & $\begin{array}{l}\text { Différence } \\
\text { haut/bas }\end{array}$ & $\mathrm{X} 70,51$ & \\
\hline
\end{tabular}

Le prix moyen d'une formation en France est de 2427,16€ tout niveau et tout domaine confondu. Cette moyenne cache une forte disparité. En effet, la médiane est à 2028€, conséquence de la présence de quelques formations onéreuses. Ainsi, la formation la moins chère coûte $156 €$ l'année tandis que la plus élevée atteint $11000 €$ soit plus de 70 fois le tarif de la première. On peut toutefois noter une certaine concentration des tarifs de formation avec une grande majorité des formations coûtant moins de $5000 €$.

\section{Graphique 7 : Effectif des formations par intervalle de prix (source base de données des FOAD françaises mars 2011)}

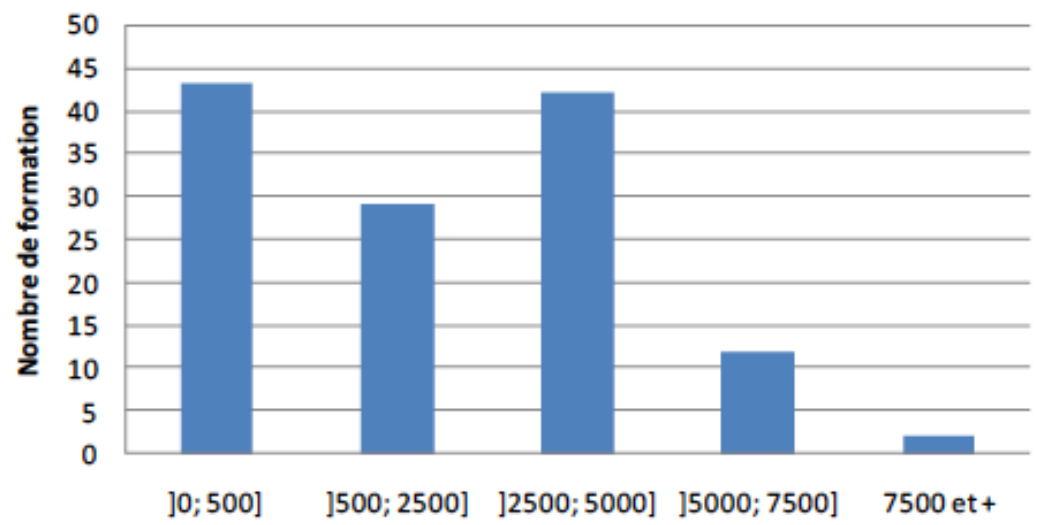

On se rend compte que $2 / 3$ des formations appartiennent à deux intervalles: celui des formations dont le prix est inférieur à $500 €$ et celui des formations dont le prix est compris entre 2500 et $5000 €$. 
Deux éléments semblent structurer le prix des formations : le niveau, la catégorie généralerecherche ou professionnelle et le domaine disciplinaire. En effet, la moyenne des prix des licences est inférieure à celle des masters : 1689,89 d'un côté et 2800,13 de l'autre. Pour ce qui est des moyennes, une formation de niveau bac +5 apparaît $40 \%$ plus cher qu'une formation à Bac+3. Dans le même temps, les diplômes généraux ou recherche ont une moyenne de prix inférieure à celle des formations professionnalisantes (licence pro et master pro) : pour les licences on est à 1125,38 les générales et 2419,21 pour les professionnelles et pour les masters nous sommes à $2225,71 €$ pour les « recherche » et $3013,23 €$ pour le master pro.

Pour ce qui est du domaine disciplinaire, les moyennes de prix apparaissent fortement différenciées. Nous avons déterminé 5 groupes de prix.

Tableau 13 : Groupes de prix des FOAD en fonction des domaines des formations en Euros (source base de données des FOAD françaises mars 2011)

\begin{tabular}{|l|l|l|}
\hline Groupes & Domaines & Prix moyens \\
\hline 1 & $\begin{array}{l}\text { lettre-langue- } \\
\text { civilisation }\end{array}$ & 886,7 \\
\hline Groupe 2 & sciences dures & 1251,34 \\
\cline { 2 - 3 } & $\begin{array}{l}\text { gestion de la nature- } \\
\text { agriculture }\end{array}$ & 1328 \\
\hline Groupe 3 & Général & 1841,57 \\
\cline { 2 - 3 } & $\begin{array}{l}\text { sciences humaines et } \\
\text { sociales }\end{array}$ & 2131,51 \\
\cline { 2 - 3 } & Education & 2425,26 \\
\hline Groupe 4 & Informatique & 3088 \\
\hline Groupe 5 & Santé & 3535,88 \\
\cline { 2 - 3 } & $\begin{array}{l}\text { Technologie/techniques } \\
- \text { entreprises }\end{array}$ & 3746,93 \\
\cline { 2 - 3 } & $\begin{array}{l}\text { économie-commerce- } \\
\text { gestion }\end{array}$ & 3896,54 \\
\hline
\end{tabular}

Il existe un écart de prix de plus de $77 \%$ entre le domaine où les prix sont les moins élevés domaine lettre-langues-civilisation - et celui où les prix sont les plus élevés -domaine économiecommerce-gestion-. Les prix les plus élevés se retrouvent au niveau des domaines santé, technologie/techniques entreprises et économie-commerce gestion. Pour rappel ce dernier domaine est celui le plus représenté dans l'offre de formation.

La FOAD française est plutôt de niveau master avec un contenu disciplinaire orienté professionnel. Le champ disciplinaire peut être difficilement applicable au monde de l'entreprise comme lettre, Langues et civilisation ou au contraire totalement intégré comme "l'économiegestion-management ».

Cette formation propose un fort tutorat dans le cadre d'une plateforme utilisant majoritairement une technologie permettant les activités asynchrones avec parfois des séances de regroupements virtuels. La plateforme utilisée est Moodle, bien qu'il y ait en général peu de communication faite sur le LMS utilisé. Ensuite, cette formation est ouverte à un large public aussi bien en formation initiale qu'en formation continue.

Le fait d'avoir moins de M1 proposés que de M2 peut s'expliquer par le fait que la formation à distance est essentiellement axée vers un public en reprise d'étude ou professionnel qui ne peut donc assurer un acte continu de formation. Il peut donc être compliqué pour ce dernier de 
s'impliquer durant deux années entières dans une formation. In fine, la formation sur un an répond plus au besoin des professionnels car plus courte et plus flexible (d'autant plus que, bien souvent, la moitié de la formation se déroule en entreprise).

L'offre de formation en e-learning en Chine se caractérise par une place importante faite aux formations des domaines économie-gestion-management et ingénierie - elles représentent à elles seules près de $70 \%$ de l'offre - et illustre bien la volonté de l'Etat chinois de favoriser un type de formation plutôt opérationnelle au service direct des territoires et des entreprises. Rappelons qu'il s'agissait pour lui de promouvoir le développement socio-économique au travers de spécialités dites « extraordinaires ».

Tout comme pour l'offre chinoise, le domaine économie-gestion arrive en tête des domaines représentés dans l'offre française, bien que ce soit en proportion plus modeste (19\%). La principale différence vient de ce que ce domaine partage la première place avec celui des lettrelangue-civilisation qui représente lui aussi 19\%. D'un côté l'offre de formation est orientée, de l'autre, en dépit des différents appels à projets, discours sur l'importance du e-learning, l'offre est laissée à l'appréciation de porteurs de projets plus ou moins soutenus par la gouvernance des établissements d'enseignement supérieur pas toujours convaincus des potentialités du elearning. Il en découle une offre de formation française qui reste somme toute anecdotique. Les mêmes effets d'annonces semblent d'ailleurs se profiler avec les MOOC. On en parle comme d'une révolution comme si auparavant rien n'avait été fait dans le domaine du e-learning en France. Il semblerait que l'Etat français s'arrête au milieu du gué en se focalisant sur l'outil, soit un mode de formation, sans qu'il n'y ait derrière une volonté stratégique d'usage de cet outil dans un but précis... « Il n’y a pas de vent favorable pour qui ne sait où il va » (Sénèque).

On avait déjà noté l'importance accordée dans l'offre française aux formations d'ordre générales, lettres langues notamment. Les formations à finalité professionnelle apparaissent moins importantes que celles présentes dans l'offre de formation chinoise.

On remarquera aussi que les formations à finalité professionnelle sont proportionnellement plus importantes en Chine qu'en France. Est-ce que cela voudrait dire que la Chine a un besoin plus important de professionnels spécialisés et diversifiés (variété plus importante qu'en France) ce qui signifierait qu'il y aurait un meilleur appariement entre formation et emploi ? et qu'en France, compte-tenu des interférences dans ce processus, les individus préféreraient se positionner sur des formations les plus larges possibles ? Cela reste en débat et mérite d'être analysé lors de travaux ultérieurs.

Pour ce qui est de la politique de mise en place du e-learning en Chine, elle semble s'illustrer par une ligne de type "top down » tant au niveau du contenu des formations que de l'usage du elearning. Elle rappelle le pilotage par « les besoins de main-d'œuvre». Tandis que celle mise place en France laisse le libre choix aux établissements de s'y engager en essayant d'orienter l'usage par des financements résultant de réponses à des appels à projet financés sur fond public. Si l'offre de formation chinoise est diversifiée et cible des compétences précises jugées importantes (formations extraordinaires) qui prend en compte les niveaux de vie différents des provinces, l'offre de formation française est peu fournie, peu diversifiée et serait en fait la résultant d'un effet « d'offre». 


\section{BIBLIOGRAPHIE}

Albero B. 2004. Technologies et formation : Travaux, interrogations, pistes de réflexion dans un champ de recherche éclaté. Savoirs 5(2):9-69.

Becker G. 1964. Human capital: A theoretical and empirical analysis, with special reference to Education

Bel M. «Formation et territoire : des approches renouvelées », Formation emploi, 97 |2007, 67-80.

Collectif. 2008. Enseignement à distance : Volonté nationale, impératif international. Distances Et Savoirs 6(1):165-8.

Feng X, Zhang W, Chen L. 2011. Distance education in rural china achieves inter-school collaboration and increased access to education. Distances Et Savoirs 9(1):53-67.

Godet M. 2007 « Manuel de prospective stratégique » Tome 1 et Tome $2-3^{\text {ème }}$ édition. Job

Gravot P. 1993. Economie de l'éducation. Paris: Economica.

Gurgand M. 2005. Économie de l'éducation. Paris: la Découverte.

Hugon P. 2005 « La scolarisation et l'éducation : facteurs de croissance ou catalyseurs du développement?», Mondes en développement 4/2005 (no 132), p. 13-28.

Jarousse J and Mingat A. 1992. La formation du capital humain : Gestion par le marché ou gestion par l'état. Revue Économique 43(4):739-54.

Mincer J. 1962. On-the-job training: Costs, returns, and some implications. Journal of Political Economy 70(5, Part 2: Investment in Human Beings):pp. 50-79.

Musselin C. , «Vers un marché international de l'enseignement supérieur ? »,Critique internationale, 2008/2 n³9, p. 13-24. DOI : 10.3917/crii.039.0013

Paul J. 2007. Economie de l'éducation. Armand Colin. Paris

Picon D. 2009. Education et richesse des nations. Sciences Humaines 205(6):40-.

Piore, M.J (1968) « On the job training and ajudjustement to technological change », Journal of Humain ressources. Vol 3 p 435-439

Plassard J and Tran nhu. 2011. Politiques publiques et régulations des systèmes d'enseignement. Toulouse: .

Poirot J. 2005. Le rôle de l'éducation dans le développement chez J. rawls et A. sen, entre équité et efficacité. Mondes En Développement 132(4):29-38. 
Psacharopoulos, G. (1994) : Return to investment in education. A global update, World Development, vol. 22, p. 1325-1343.

Tanguy L. (dir) 1986. L’introuvable relation formation-emploi. La Documentation Française

Tremblay G. 2009. La formation à distance en contexte de globalisation. Distances Et Savoirs 7(4):715-31.

Wauthy X. 2006. Université et concurrence : Quelques apports théoriques récents. Reflets Et Perspectives De La Vie Économique XLV(2):31-8.

Whalen T and Wright D. 1999. Methodology for cost-benefit analysis of web-based tele-learning: Case study of the bell online institute. American Journal of Distance Education 15(1):24-44.

Yin RK. 2003.

Case study research, design and methods. 3rd ed. . Newbury Park, CA: Sage Publications.

Zawacki-Richter O, Backer EM, Vogt S. 2009. Review of distance education research (2000 to 2008): Analysis of research areas, methods, and authorship patterns. International Review of Research in Open and Distance Learning 10(6).

\section{ANNEXES}

\section{Liste des abréviations des universités chinoises et classement en étoile.}

\begin{tabular}{|c|c|c|}
\hline Classement & Abreviation. & Denomination d' universities \\
\hline \multirow{2}{*}{$\star \star \star \star \star \star ~$} & Tsinghua & Tsinghua University \\
\hline & PKU & Peking University \\
\hline \multirow{18}{*}{$\star \star \star \star \star \star ~$} & BNU & Beijing Normal University \\
\hline & BAAU & Beijing University of Aeronautics \&astronautics \\
\hline & RUC & Renmin University of China \\
\hline & CAU & China Agricultural University \\
\hline & CCOM & Central Conservatory of Music \\
\hline & HIT & Harbin Institute of Technology \\
\hline & JLU & Jilin University \\
\hline & TJU & Tianjin University \\
\hline & Nankai & Nankai University \\
\hline & SDU & Shandong University \\
\hline & SJTU & Shanghai Jiaotong University \\
\hline & FUDAN & Fudan University \\
\hline & Tongji & Tongji University \\
\hline & ECUST & East China University of Science and Technology \\
\hline & ZJU & Zhejiang University \\
\hline & XMU & Xiamen University \\
\hline & USTC & University of Science and Technology of China \\
\hline & HUST & Huazhong University of Science and Technology \\
\hline
\end{tabular}




\begin{tabular}{|c|c|c|}
\hline & WHU & Wuhan University \\
\hline & CSU & Central South University \\
\hline & SYSU & Sun Yan-Sen University \\
\hline & SCU & Sichuan University \\
\hline & XJTU & Xi'an Jiaotong University \\
\hline & NJU & Nanjing University \\
\hline \multirow{22}{*}{$\star \star \star \star ~$} & BUPT & Beijing University of Posts and Telecommunications \\
\hline & BIT & Beijing Institute of Technology \\
\hline & BJU & Beijing Jiaotong University \\
\hline & USTB & University of Science \&Technology Beijing \\
\hline & BJPEU & China University of Petroleum(Beijing) \\
\hline & CUG & China University of Geosciences)(Pekin) \\
\hline & NENU & Northeast Normal University \\
\hline & DLUT & Dlian University of Technology \\
\hline & NEU & Northeastern University \\
\hline & UPC & China University of Petroleum(east) \\
\hline & ECNU & East China Normal University \\
\hline & CCNU & Central China Normal University \\
\hline & CUG & China University of Geosciences（Wuhan） \\
\hline & HNU & Hunan University \\
\hline & SCUT & South China University of Technology \\
\hline & CQU & Chongqin University \\
\hline & SWJTU & Southwest Jiaotong University \\
\hline & UESTC & University of Electronic Science and Technology of China \\
\hline & NWPU & Northwestern Polytechnical University \\
\hline & XIDIAN & Xidian University \\
\hline & $\mathbf{L Z U}$ & Lanzhou University \\
\hline & SEU & Southeast University \\
\hline \multirow{10}{*}{$\star \star \star$} & BFSU & Beijing Foreign Studies University \\
\hline & BUCM & Beijing University of Chinese Medicine \\
\hline & CMU & China Medical University \\
\hline & DHU & Donghua University \\
\hline & SHISU & Shanghai International Studies University \\
\hline & $\mathbf{Z Z U}$ & Zhenzhou University \\
\hline & WHUT & Wuhan Universiyu of Technology \\
\hline & SCNU & South China Normal University \\
\hline & SWNU & Southwest University \\
\hline & SYTU & Jiangnan University \\
\hline \multirow{11}{*}{$<\star \star \star$} & BLCU & Beijing Language and Culture University \\
\hline & CUC & Communication University of China \\
\hline & UIBE & University of International of Business and Economics \\
\hline & NEAU & Northeast Agricultural University \\
\hline & DUFE & Donbei University of Finance and Economics \\
\hline & FJTU & Fujian Normal University \\
\hline & SWUST & Southwest University of Science and Technology \\
\hline & SWUFE & Southwestern University of Finance and Economics \\
\hline & SICAU & Sichuan Agricultural University \\
\hline & SNNU & Shaanxi Normal University \\
\hline & OUC & The Open University of China \\
\hline
\end{tabular}


